\title{
A família dos poliedros fulerenos
}

\author{
Roberto Ribeiro Paterlini(D)
}

\begin{abstract}
Resumo
Nos últimos 40 anos, as moléculas denominadas fulerenos têm sido extensivamente estudadas em várias ciências, particularmente na Química. Como os poliedros constituem modelos dessas moléculas, vemos uma oportunidade para o professor de Matemática do Ensino Médio praticar a interdisciplinaridade e, ao mesmo tempo, incentivar o estudo da Geometria Espacial junto a seus estudantes. No presente texto pretendemos fornecer ao professor subsídios para essa prática.
\end{abstract}

Palavras-chave: Fulerenos combinatórios; lei dos doze pentágonos; modelos de fulerenos; fulerenostubo; poliedros de Goldberg.

\section{Abstract}

In the last 40 years, molecules called fullerenes have been extensively studied in various sciences, particularly in Chemistry. As polyhedra are models of these molecules, we see an opportunity for the Mathematics teacher at High School to practice interdisciplinarity and, at the same time, encourage the study of Spatial Geometry by their students. In this text we intend to provide the teacher with subsidies for this practice.

Keywords: Combinatorial fullerenes; law of the twelve pentagons; fullerene models; tube fullerenes; Goldberg polyhedra.

\section{Introdução}

Os fulerenos são estruturas moleculares formadas exclusivamente por átomos de carbono, organizados em uma forma poliédrica com faces pentagonais e hexagonais apenas, e de modo que cada átomo liga-se a outros três. As faces não são necessariamente polígonos regulares.

Neste texto pretendemos apresentar ao professor de Matemática do Ensino Médio uma breve explanação sobre a presença dessas estruturas na Química e, principalmente, estudar suas propriedades geométricas mais elementares. Pensamos assim incentivar o professor a praticar a interdisciplinaridade com essa fantástica ciência, a Química, e, ao mesmo tempo, prover-se de sequências didáticas que possam estimular o interesse dos seus estudantes para a Geometria Espacial, especialmente para o estudo dos poliedros.

Alertamos que, na literatura especializada, a definição de fulereno tem variações. Por exemplo, a definição proposta por Melker e Kuprina [9] não exige que as faces sejam exclusivamente pentagonais ou hexagonais. No presente texto adotamos a definição da maioria dos autores que consultamos, como a de Buchstaber e Erokhovets [1], pág. 24. 


\section{Exemplos de fulerenos}

O exemplo mais imediato de fulereno é a estrutura $\mathrm{C}_{20}$, constituída por 20 átomos de carbono. Na Figura 1 vemos uma representação dessa estrutura, e o leitor pode reconhecer que seu modelo geométrico, conforme desenhado na Figura 2, é o dodecaedro com faces pentagonais. Uma aresta entre dois vértices indica que os átomos correspondentes estão quimicamente ligados. Esse poliedro tem 20 vértices, 30 arestas e 12 faces. Cada vértice é do tipo três, isto é, a ele concorrem três arestas (ou três faces). Uma molécula que tem como base essa estrutura é o dodecaedrano $\mathrm{C}_{20} \mathrm{H}_{20}$, um hidrocarboneto. Nessa molécula cada átomo de carbono está ligado a outros três átomos de carbono e a um átomo de hidrogênio. Para mais informações sobre o dodecaedrano consulte [18]. O fulereno $\mathrm{C}_{20}$ (Figura 1) é muito reativo e, portanto, difícil de ser obtido e isolado. Em 2000, ele foi obtido em fase gasosa a partir do dodecaedrano (Prinzbach et al. [13]).

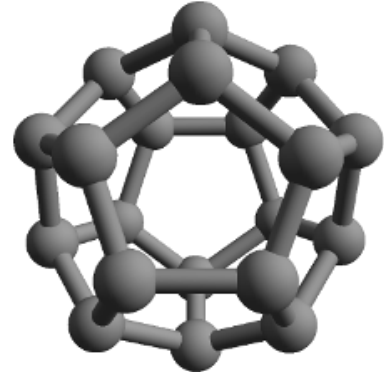

Figura 1: Organização dos átomos de carbono na estrutura do fulereno $\mathrm{C}_{20}$. Figura da Wikipedia [27].

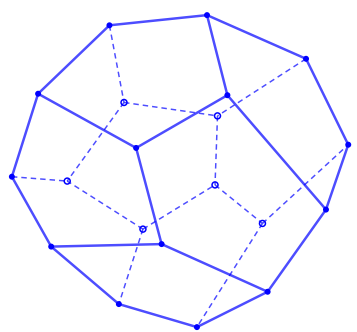

Figura 2: Representação poliédrica (sem escala correta) da estrutura $\mathrm{C}_{20}$.

Segundo Parasuk e Amlöf [10], pág.188, a representação geométrica da estrutura $\mathrm{C}_{20}$ é um dodecaedro, mas não regular. As distâncias entre os átomos, representadas pelos comprimentos das arestas, são 1,416, 1,425 e 1,474 angstroms. Um angstrom é uma unidade de comprimento equivalente a $10^{-10}$ metros. Esses valores repetem-se simetricamente nas arestas, formando faces congruentes duas a duas. No desenho da Figura 2, a representação está fora de escala. Uma possibilidade de modelo é o tetartoide. Veremos, mais adiante, como construir modelos desse poliedro.

Depois do $\mathrm{C}_{20}$, a estrutura de fulereno mais comumente considerada é o $\mathrm{C}_{60}$. Um modelo é o icosaedro truncado, muito conhecido e estudado, pelo menos desde o tempo de Arquimedes, c. de 250 a.C. Esse poliedro tem 60 vértices, 90 arestas e 32 faces, sendo 12 pentagonais e 20 hexagonais. Se essas faces forem regulares, esse poliedro é um dos sólidos semirregulares de Arquimedes. Confira, na Figura 3, uma representação.

Do ponto de vista da Arquitetura, estruturas como a do icosaedro truncado são utilizadas para construir cúpulas desde tempos antigos. São os chamados domos geodésicos e, modernamente, foram popularizados pelo arquiteto estadunidense R. Buckminster Fuller (1895-1983), que projetou um domo gigantesco para o pavilhão norte-americano da Exposição Mundial de 1967, realizada em Montreal, Canadá. Consulte [21] para ler mais detalhes.

Por muito tempo os químicos especularam sobre a existência de moléculas contendo a estrutura $\mathrm{C}_{60}$. Não se conhecia nenhum composto de que ela fizesse parte. Depois do esforço de muitos pesquisadores, os químicos Robert Curl, Harold Kroto e Richard Smalley conseguiram, em 1985, sintetizar essa estrutura como uma molécula, reconhecendo-a como o $\mathrm{C}_{60}$. Eles a chamaram 
de buckminsterfulereno em homenagem ao arquiteto referido acima. Devido a tal feito, esses pesquisadores receberam o prêmio Nobel de Química de 1996.

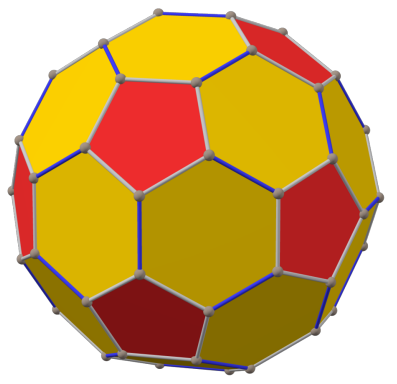

Figura 3: Representação do icosaedro truncado semirregular. Figura da Wikipedia [19].

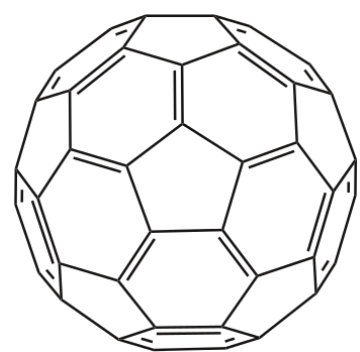

Figura 4: Representação poliédrica do $\mathrm{C}_{60}$, mostrando as ligações duplas. Figura da Wikipedia [20].

A Figura 4 exibe um desenho do $\mathrm{C}_{60}$ no qual as faces estão distribuídas como no icosaedro truncado (veremos que outras distribuições são possíveis). Observe que, em nosso desenho, em cada hexágono existem três ligações duplas alternando com ligações simples. Dessa forma a valência de cada átomo de carbono é 4. Nossas figuras, por comodidade, usam polígonos regulares. Mas, segundo informam Schwerdtfeger e outros [16], nas moléculas as ligações simples têm comprimentro de 1,442 angstroms, e as duplas, 1,378 angstroms. Uma pequena diferença difícil de expressar nos desenhos.

\section{Propriedades combinatórias dos fulerenos}

A partir desta seção denominamos fulerenos combinatórios, ou simplesmente fulerenos, aos poliedros formados por pentágonos ou hexágonos, e cujos vértices são do tipo três, isto é, a cada um concorrem três arestas. Além disso exigimos que o poliedro seja topologicamente uma esfera, o que significa que os vértices e arestas podem ser projetados sobre a superfície de uma esfera que os envolva sem que a estrutura combinatória das faces, arestas e vértices seja modificada. Um fulereno com n vértices será anotado por $\mathrm{C}_{\mathrm{n}}$.

Com essa definição sinalizamos que estamos interessados no estudo combinatório dessa família de poliedros e, apenas eventualmente, suas propriedades químicas ou métricas serão consideradas. A definição básica de poliedro que adotamos é a que está apresentada em [12], seção 19.5.

Iniciamos nossas investigações sobre propriedades geométricas dos fulerenos com uma observação induzida dos dois exemplos acima apresentados, o dodecaedro e o icosaedro truncado: a quantidade de pentágonos, nos dois casos, é 12. Será esse um comportamento recorrente nos fulerenos? Propomo-nos começar por aí.

Conforme é costume no estudo dos poliedros, dado um deles, indicaremos por $\mathrm{V}$ a quantidade de seus vértices, por A, a de suas arestas, e por F, a de suas faces. A relação de Euler é a principal fórmula combinatória dos poliedros que são topologicamente uma esfera:

$$
\mathrm{V}-\mathrm{A}+\mathrm{F}=2
$$


Usaremos mais duas fórmulas combinatórias, ambas muito simples. Contando as arestas pelos vértices, como a cada vértice concorrem três arestas temos, a princípio, 3V arestas. Mas nesse processo as arestas foram contadas em dobro, pois cada aresta está ligada a dois vértices. Obtemos a fórmula

$$
2 \mathrm{~A}=3 \mathrm{~V}
$$

válida apenas no nosso caso, em que os vértices são todos do tipo três.

Contando agora as arestas usando as faces, vemos que cada face pentagonal contribui com 5 arestas e cada face hexagonal, com 6 . Chamamos, conforme é costume, a quantidade de faces pentagonais de $\mathrm{F}_{5}$, e, a de hexagonais, de $\mathrm{F}_{6}$. Como cada aresta pertence a duas faces, temos a fórmula

$$
2 \mathrm{~A}=5 \mathrm{~F}_{5}+6 \mathrm{~F}_{6}
$$

válida apenas no nosso caso, em que as faces são pentágonos ou hexágonos.

Temos de imediato nosso primeiro resultado:

Teorema 1 (Lei dos doze pentágonos). Em qualquer fulereno, a quantidade de faces pentagonais é 12.

Demonstração. Começando com a fórmula (1) e, em seguida, introduzindo (2), temos

$$
\mathrm{V}-\mathrm{A}+\mathrm{F}=2 \Rightarrow 3 \mathrm{~V}-3 \mathrm{~A}+3 \mathrm{~F}=6 \Rightarrow 2 \mathrm{~A}-3 \mathrm{~A}+3 \mathrm{~F}=6 \Rightarrow 3 \mathrm{~F}=6+\mathrm{A}
$$

Multiplicando por 2 temos $6 \mathrm{~F}=12+2 \mathrm{~A}$. Introduzindo $\mathrm{F}=\mathrm{F}_{5}+\mathrm{F}_{6}$ e (3) nessa última relação, tem-se $6\left(\mathrm{~F}_{5}+\mathrm{F}_{6}\right)=12+5 \mathrm{~F}_{5}+6 \mathrm{~F}_{6}$, o que implica

$$
\mathrm{F}_{5}=12
$$

Se o poliedro tiver vértices de tipos diferentes de três, não vale, necessariamente, o resultado do Teorema 1. Um exemplo é o icositetraedro pentagonal, um dos sólidos de Catalan. Ele tem 38 vértices, 60 arestas e 24 faces, todas pentagonais (não regulares). Existem 32 vértices do tipo três e 6 do tipo quatro. Para mais detalhes consulte [22].

A fórmula (4) simplifica bastante algumas relações nos fulerenos. Por exemplo, podemos obter os valores $\mathrm{n}=\mathrm{V}$, A e $\mathrm{F}$ em função de $\mathrm{F}_{6}$ apenas:

Corolário 1. Em qualquer fulereno $\mathrm{C}_{\mathrm{n}}$ temos

$$
\mathrm{n}=\mathrm{V}=2\left(10+\mathrm{F}_{6}\right) \quad \mathrm{A}=3\left(10+\mathrm{F}_{6}\right) \quad \mathrm{F}=12+\mathrm{F}_{6}
$$

Demonstração. Usando a fórmula (3) temos $2 \mathrm{~A}=5 \mathrm{~F}_{5}+6 \mathrm{~F}_{6} \Rightarrow 2 \mathrm{~A}=5 \cdot 12+6 \mathrm{~F}_{6}=60+6 \mathrm{~F}_{6} \Rightarrow$ $\mathrm{A}=30+3 \mathrm{~F}_{6}=3\left(10+\mathrm{F}_{6}\right)$.

Por outro lado, a fórmula (2) dá-nos $3 \mathrm{~V}=2 \mathrm{~A}=60+6 \mathrm{~F}_{6} \Rightarrow \mathrm{V}=20+2 \mathrm{~F}_{6}=2\left(10+\mathrm{F}_{6}\right)$.

Finalmente, $\mathrm{F}=\mathrm{F}_{5}+\mathrm{F}_{6}=12+\mathrm{F}_{6}$, e terminamos a verificação de (5). 
Dado um fulereno $\mathrm{C}_{\mathrm{n}}$ com valor $\mathrm{n}$ especificado, já podemos calcular seus valores $\mathrm{V}, \mathrm{A}, \mathrm{F}, \mathrm{F}_{5}$ e $\mathrm{F}_{6}$ em função de n:

Corolário 2. Dado um fulereno $\mathrm{C}_{\mathrm{n}}$ temos:

$$
\mathrm{V}=\mathrm{n} \quad \mathrm{A}=\frac{3 \mathrm{n}}{2} \quad \mathrm{~F}=\frac{\mathrm{n}}{2}+2 \quad \mathrm{~F}_{5}=12 \quad \mathrm{~F}_{6}=\frac{\mathrm{n}}{2}-10
$$

Demonstração. Temos $\mathrm{V}=\mathrm{n}$ devido à definição de $\mathrm{n}$ na notação $\mathrm{C}_{\mathrm{n}}$, e $\mathrm{F}_{5}=12$ já foi obtido. De (5) vem $\mathrm{V}=2\left(10+\mathrm{F}_{6}\right) \Rightarrow \mathrm{n}=2\left(10+\mathrm{F}_{6}\right) \Rightarrow \mathrm{F}_{6}=\frac{\mathrm{n}}{2}-10 \Rightarrow \mathrm{F}=\frac{\mathrm{n}}{2}-10+12 \Rightarrow \mathrm{F}=\frac{\mathrm{n}}{2}+2$.

Por outro lado, ainda de $(5)$, temos $A=3\left(10+F_{6}\right)=3\left(10+\frac{n}{2}-10\right) \Rightarrow A=\frac{3 n}{2}$.

São úteis as seguintes observações:

Corolário 3. Dado um fulereno $\mathrm{C}_{\mathrm{n}}$ temos:

$$
\mathrm{n} \text { é par } \quad e \quad \mathrm{n} \geq 20
$$

Demonstração. Do Corolário 2 temos $\mathrm{F}=\frac{\mathrm{n}}{2}+2$, o que implica que $\mathrm{n}$ é par. Ainda, como $\mathrm{F} \geq 12$, temos $\mathrm{n}=2 \mathrm{~F}-4 \geq 2 \cdot 12-4=20$.

Precisamos agora determinar para que valores de $n$ existem fulerenos $C_{n}$. Começamos com

Teorema 2. Para todo inteiro $\mathrm{p} \geq 0$ com $\mathrm{p} \neq 1$, existem fulerenos com $\mathrm{F}_{6}=\mathrm{p}$.

Aqui a demonstração é mais técnica. Faremos alguns comentários no final deste texto. Completamos com o

Corolário 4. Existem fulerenos (combinatórios) $\mathrm{C}_{\mathrm{n}}$ para valores $\mathrm{n}=20$ e todo inteiro par $\mathrm{n} \geq 24$.

Demonstração. No Corolário 2 vimos que $\mathrm{n}=2\left(10+\mathrm{F}_{6}\right)$. Se $\mathrm{F}_{6}=0$, segue $\mathrm{n}=20$. Se $\mathrm{F}_{6} \geq 2$, vem $\mathrm{n}=20+2 \mathrm{~F}_{6} \geq 24$ e n é par e, devido ao Teorema 2 , n pode ser qualquer par $\geq 24$.

Determinamos assim que, do ponto de vista geométrico-combinatório, existem fulerenos que modelam cada uma das estruturas $\mathrm{C}_{20}, \mathrm{C}_{24}, \mathrm{C}_{26}, \mathrm{C}_{28}, \ldots$ e, para cada um deles, sabemos os valores de V, A e F, distinguindo a quantidade de pentágonos (que sempre é 12) e a de hexágonos. Entretanto, em geral, uma estrutura $\mathrm{C}_{\mathrm{n}}$ tem mais de um poliedro como modelo, pois pode variar a distribuição das faces. Veremos exemplos mais adiante.

\section{Construção de modelos de fulerenos}

Nesta seção pretendemos, principalmente, prover o professor de Matemática de elementos suficientes para construir sequências didáticas nas quais seus estudantes possam investigar os fulerenos por meio de modelos. Esses modelos podem ser construídos com papel-cartão, varetas ou com outro método. Para facilitar podemos, sempre que possível, usar polígonos regulares como faces. Observamos, entretanto, que a maioria dos fulerenos não pode ter todas as faces regulares. Em certas situações, na construção de modelos, devemos inclusive permitir que as faces sejam não planas. Com isso evitamos fazer muitos cálculos para determinar as medidas das faces. Lembramos 
que são as propriedades combinatórias que mais nos interessam, e, em menor grau, as propriedades métricas.

Nas figuras 1 e 2 vimos representações da estrutura $\mathrm{C}_{20}$. O dodecaedro regular é bem conhecido dos estudantes e, em muitos laboratórios escolares de Geometria, vemos modelos construídos por eles. No contexto do presente estudo, entretanto, um bom modelo da estrutura $\mathrm{C}_{20}$ precisa ter arestas com três medidas diferentes, conforme foi comentado. Um dodecaedro com faces pentagonais que possui essa característica é o tetartoide, um poliedro com conexões com a Mineralogia.

Usando informações de [23], as faces do tetartoide são congruentes duas a duas e cada uma tem arestas com três medidas, digamos a, b e c, sendo uma aresta de medida c, duas consecutivas de medida b e duas consecutivas de medida a. Ao organizar as faces para montar o poliedro ocorrem vértices com a seguinte distribuição:

4 vértices aos quais concorrem três arestas de medida a;

4 vértices aos quais concorrem três arestas de medida b;

12 vértices aos quais concorrem três arestas, uma de cada medida.

Por outro lado, como existem 12 faces e as arestas de medida c, uma em cada face, são comuns a duas faces, existem 6 dessas arestas. Do mesmo modo, vê-se que existem 12 arestas de medida a e 12 de medida b.

Temos, na Figura 5, o modelo de uma face de um tetartoide, que pode ser desenhado com um aplicativo para Geometria. Na Figura 6 vemos um desenho indicativo do poliedro para mostrar como devem ser combinadas as faces. Na Figura 7 temos a foto de um modelo. As diferenças entre as medidas das arestas estão exageradas em relação àquelas correspondentes do fulereno $\mathrm{C}_{20}$.

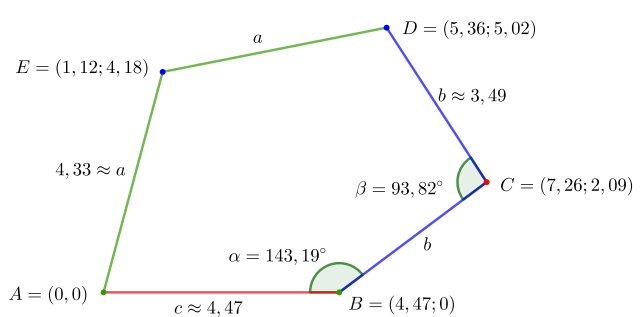

Figura 5: Face de um tetartoide.

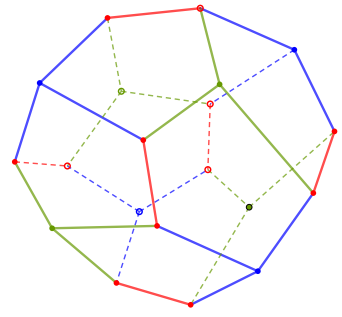

Figura 6: Desenho de um tetartoide. 
Figura 7. Modelo de tetartoide construído em papel-cartão pelo autor. O modelo está pousado em uma aresta de cor vermelha, em posição similar à do desenho da Figura 6. As cores das arestas distinguem as diferentes medidas. Foto do autor.

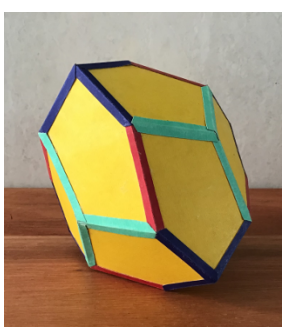

Na Figura 8 vemos o desenho de um poliedro $\mathrm{C}_{24}$, e, na Figura 9, desenho da esquerda, uma "meia" planificação. Trata-se de um fulereno com 12 faces pentagonais e 2 hexagonais, portanto, $\mathrm{F}=14$. Temos ainda $\mathrm{V}=24$ e $\mathrm{A}=36$. O desenho do poliedro foi adaptado de Talanov e outros [17], pág. 359.

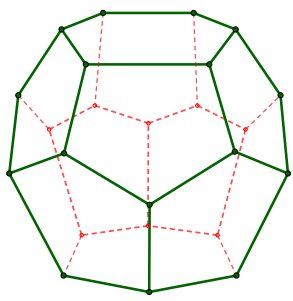

Figura 8: Poliedro $\mathrm{C}_{24}$.
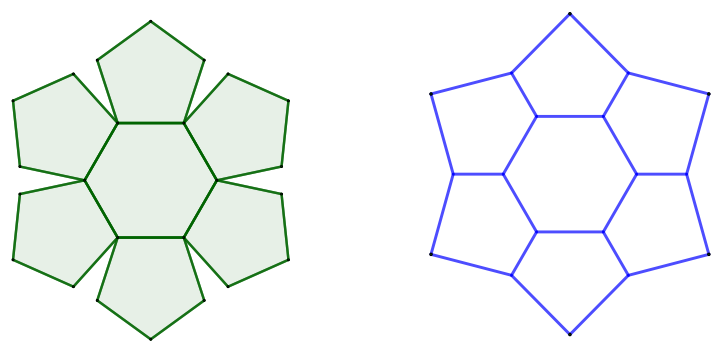

Figura 9: "Meia" planificação (indicativa) do poliedro $\mathrm{C}_{24} \mathrm{e}$ grafo correspondente.

Na Figura 9, à direita, temos um grafo, um desenho representativo da projeção dessa parte do poliedro sobre o plano que contém a face hexagonal. O grafo informa a estrutura combinatória do poliedro, mas as medidas das arestas não são necessariamente iguais às correspondentes do poliedro.

Figura 10. Modelo do $\mathrm{C}_{24}$ construído em papel-cartão pelo autor. Por facilidade, as faces foram recortadas como polígonos regulares. Devido a isso ocorrem pequenas distorções. O modelo está pousado em uma face hexagonal, em posição similar à do desenho da Figura 8.

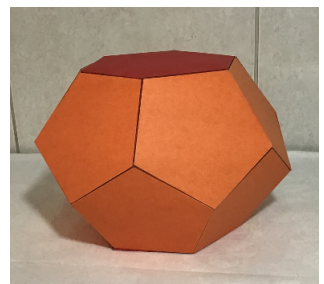


A Figura 11 exibe o desenho de um poliedro $\mathrm{C}_{26}$. Trata-se de um fulereno com 12 faces pentagonais e 3 hexagonais, portanto, $\mathrm{F}=15$. Temos ainda $\mathrm{V}=26$, todos de tipo três, e $\mathrm{A}=39$. Na Figura 12 vemos a foto de um modelo. Uma planificação pode ser vista na Figura 13.

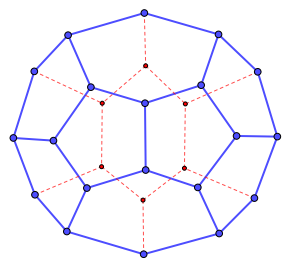

Figura 11: Desenho do poliedro $\mathrm{C}_{26}$.

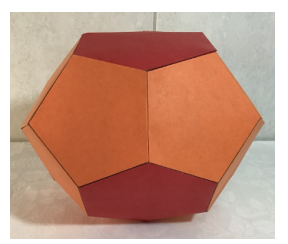

Figura 12: Modelo do poliedro $\mathrm{C}_{26}$. O poliedro está pousado em uma aresta, em posição similar à da Figura 11. Construído em papel-cartão pelo autor. Foto do autor.

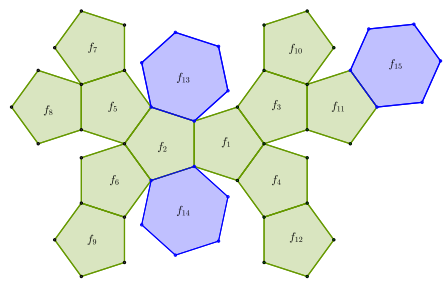

Figura 13: Uma planificação (indicativa) do poliedro $\mathrm{C}_{26}$.

Na Figura 14 vemos o desenho de um poliedro representante da estrutura $\mathrm{C}_{28}$. Ele tem 12 faces pentagonais e 4 hexagonais, portanto, $\mathrm{F}=16$. Temos ainda $\mathrm{V}=28$, todos de tipo três, e $\mathrm{A}=42$. A Figura 15 traz uma foto de um modelo do mesmo poliedro.

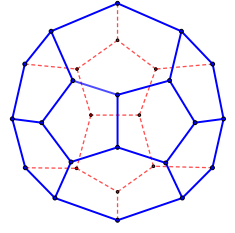

Figura 14: Um poliedro $\mathrm{C}_{28}$.

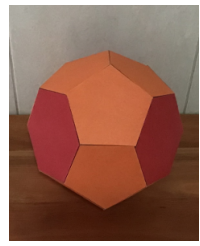

Figura 15: Modelo do poliedro da Figura 14. Ele está em posição similar à do desenho, mas com um giro horizontal de $180^{\circ}$. Construído em papelcartão pelo autor. Foto do autor. 
Representações poliédricas das estruturas $\mathrm{C}_{24}, \mathrm{C}_{26}$ e $\mathrm{C}_{28}$ não podem ter, por razões teóricas, todas as faces regulares. Mas, para certas finalidades pedagógicas, é possível construir os modelos dessa forma pois as distorções são pequenas. Com isso evitamos muitos cálculos para determinar os comprimentos das arestas e as medidas dos ângulos das faces. No final do texto faremos observações mais detalhadas sobre essa questão.

Explicamos agora como construir modelos de fulerenos-tubo. Em Química, muitos os chamam "nanotubos fechados".

A representação clássica de um nanotubo consiste de um cilindro circular reto no qual são dispostos os vértices de hexágonos de forma que, quando são projetadas as arestas sobre a superfície cilíndrica, essas formas hexagonais perfazem um ladrilhamento. Em um nanotubo fechado, a parte cilíndrica é completada com duas capas topologicamente em forma de semiesferas, constituídas por 6 pentágonos cada uma e por uma quantidade de hexágonos que varia com o formato escolhido. Existem outras propostas de representação poliédrica para o nanotubo; confira, por exemplo, Cox e Hill [3].

Na Figura 16 temos uma planificação de um fulereno-tubo, separada em três partes, com a finalidade de facilitar nossa exposição. No desenho da esquerda vemos faces hexagonais organizadas em três fileiras com seis hexágonos cada. Essas faces devem ser dobradas de forma que a face $\mathrm{h}_{1}$ tenha uma aresta em comum com cada uma das faces $h_{6}$ e $h_{12}$, a face $h_{12}$ tenha uma aresta em comum, além de $h_{1}$, com cada uma das faces $h_{7}$ e $h_{13}$, e essa tenha uma aresta comum também com $h_{18}$. Com isso forma-se uma estrutura em que os vértices estão dispostos na superfície de um cilindro.
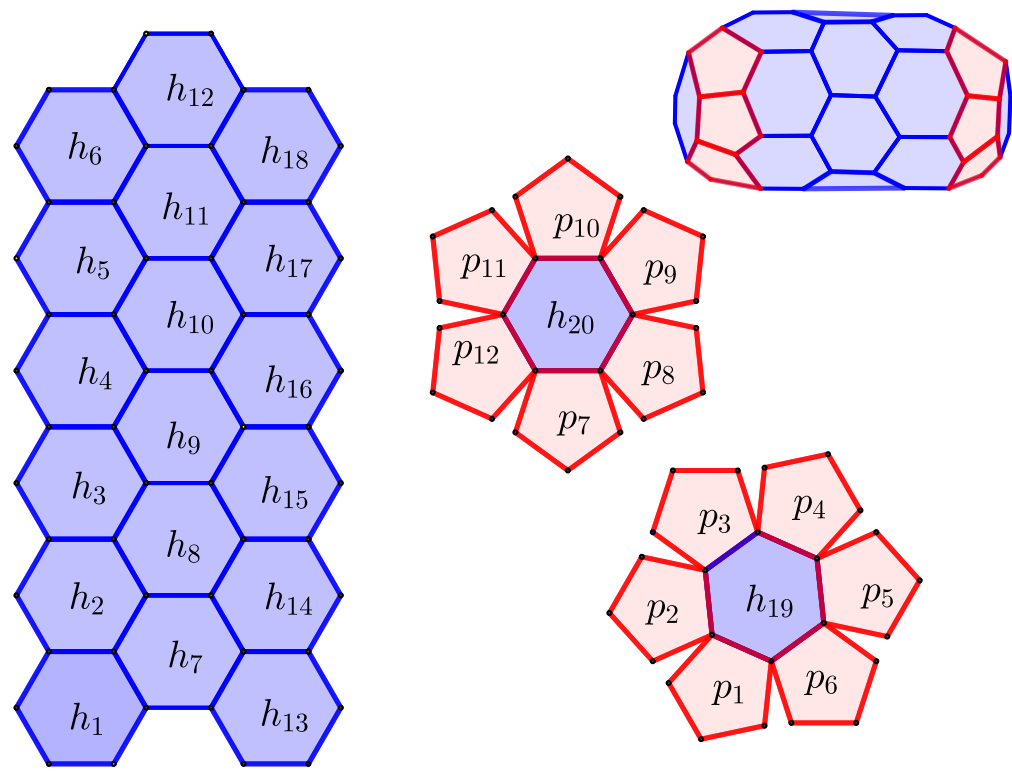

Figura 16: Uma planificação (indicativa) do fulereno-tubo $\mathrm{C}_{60}$ com desenho do modelo.

No desenho da direita, ainda na Figura 16, vemos duas cópias idênticas de um hexágono cercado 
por seis pentágonos. Depois de recortadas, em cada uma delas juntamos as arestas laterais dos pentágonos, formando duas capas. Essas capas juntam-se ao tubo, uma em cada ponta, para formar um poliedro. Ainda na Figura 16 vemos um desenho do poliedro.

Observe que esse fulereno-tubo tem 12 pentágonos e 20 hexágonos, portanto, a mesma quantidade de faces de cada tipo que o icosaedro truncado. Trata-se de um segundo modelo da estrutura $\mathrm{C}_{60}$, com as faces distribuídas de forma diferente.

Para construir a parte cilíndrica do fulereno-tubo $\mathrm{C}_{60}$ usamos três anéis com seis hexágonos cada. Variando a quantidade de anéis podemos construir uma infinidade de fulerenos tubos. Usando as fórmulas da Seção 2 vemos que com p anéis, $\mathrm{p} \geq 1$, temos um fulereno-tubo $\mathrm{C}_{24+12 \mathrm{p}}$.

As capas dos fulerenos-tubo podem ter outras formas. Por exemplo, na Figura 17 vemos como modelar capas usando "meio" icosaedro truncado. Para construir uma dessas capas, iniciamos com uma face pentagonal desse poliedro, e tomamos os dois primeiros anéis que o rodeiam. O primeiro anel é constituído de cinco faces hexagonais, e o segundo de dez faces, que se alternam entre faces pentagonais e faces hexagonais. A parte cilíndrica é modelada com cintas de dez hexágonos cada. Observe que a posição dos hexágonos nessas cintas é diferente daquela da Figura 16.
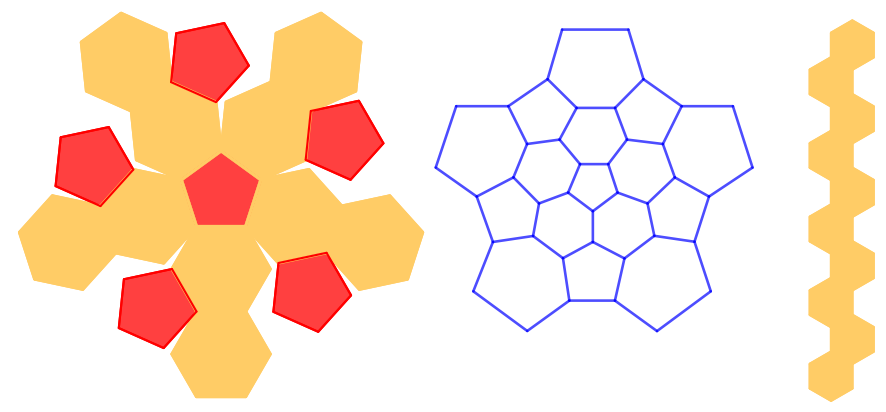

Figura 17: Uma planificação (indicativa) de "meio" icosaedro truncado, desenho do grafo correspondente, e uma planificação mostrando uma cinta de dez hexágonos.

Usando capas de "meio" icosaedro truncado e p cintas com dez hexágonos cada, fabricamos modelos em forma de tubos das estruturas $\mathrm{C}_{60+20 \mathrm{p}}$. Se $\mathrm{p}=1$ (uma cinta) temos, um fulereno tubo $\mathrm{C}_{80}$ (Figura 18), se $\mathrm{p}=2$ (duas cintas) temos um fulereno tubo $\mathrm{C}_{100}$ (Figura 20), e se $\mathrm{p}=3$ (três cintas) temos um fulereno tubo $\mathrm{C}_{120}$ (Figura 19).

Lembramos que esses fulerenos-tubo não podem ter todas as faces regulares. Esses poliedros não fazem parte das listas de poliedros convexos com faces regulares, conforme se pode consultar a partir de [24]. Portanto nossos desenhos e modelos são indicados para fins pedagógicos no ensino fundamental. Usando varetas maleáveis é possível fazer belos modelos. Confira, por exemplo, Hart [8], pág. 136. 


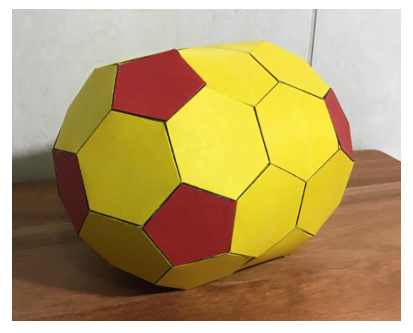

Figura 18: Modelo do fulereno-tubo $\mathrm{C}_{80}$. Construído em papel-cartão pelo autor. Foto do autor.

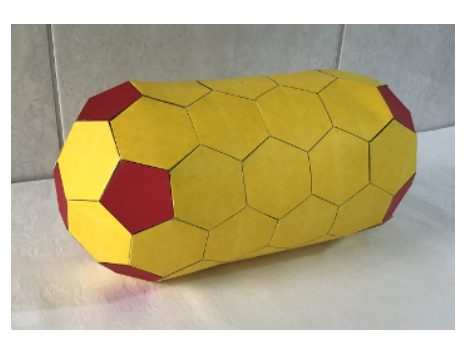

Figura 19: Modelo do fulereno-tubo $\mathrm{C}_{120}$. Construído em papel-cartão pelo autor. Foto do autor.

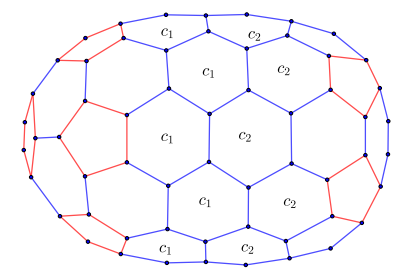

Figura 20: Desenho do fulereno-tubo $\mathrm{C}_{100}$. Cada uma das capas é um "meio" icosaedro truncado. O tubo é formado por duas cintas $c_{1}$ e $c_{2}$.

Sobre esse assunto de construção de fulerenos através de modelos, completamos esta seção comentando que poliedros convexos com faces pentagonais e hexagonais foram estudados por Michael Goldberg (1902-1990), em 1937. Na respectiva página da Wikipedia [25] podemos ver belos desenhos desses poliedros, como o da Figura 21. As faces pentagonais são regulares e as hexagonais são equiláteras.

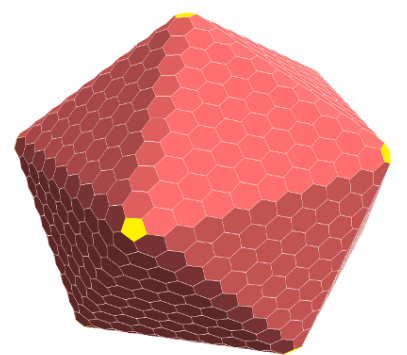

Figura 21: Um poliedro fulereno de Goldberg. Figura da Wikipedia [25]. 
Mais exatamente, os poliedros de Goldberg são, por definição, convexos, têm faces pentagonais e hexagonais apenas, todos os vértices são do tipo três, e têm simetria icosaédrica. Na Nota 3 apresentaremos explicações sobre essas simetrias.

Todo poliedro de Goldberg é um fulereno, mas nem todo fulereno é um poliedro de Goldberg, mesmo se for convexo. Em [6], Goldberg determinou que, nesses poliedros, a quantidade de vértices é $\mathrm{V}=20\left(\mathrm{a}^{2}+\mathrm{ab}+\mathrm{b}^{2}\right)$, em que $0 \leq \mathrm{b} \leq \mathrm{a}$ são inteiros, com $\mathrm{a}>0$. Portanto, existem poliedros de Goldberg representantes das estruturas $C_{n}$ para $n=20,60,80,140,180,240$, etc. Para esses poliedros temos ainda $F=10\left(a^{2}+a b+b^{2}\right)+2$ e $A=30\left(a^{2}+a b+b^{2}\right)$.

Construir um modelo do poliedro de Goldberg da Figura 21 certamente exige muita paciência. Modelos com menos faces são o dodecaedro e o icosaedro truncado, já citados. Outro que pode ser construído é o triacontaedro rômbico truncado (TRT), que corresponde aos valores a $=2$ e b $=0$ nas fórmulas acima. A quantidade de vértices é 80, portanto o TRT é um modelo da estrutura $\mathrm{C}_{80}$. Ele tem 42 faces, sendo 12 pentagonais regulares e 30 hexagonais equiláteras. A quantidade de arestas é 120 .

O poliedro TRT, representado na Figura 22, pode ser obtido, como o nome diz, por truncamento do triacontaedro rômbico, com os cortes executados apenas nos 12 vértices de tipo 5. Cada um desses 12 vértices dá origem a uma face pentagonal. O corte pode ser feito de forma que essas faces sejam regulares e as faces restantes sejam hexágonos equiláteros. O ângulo do hexágono, em dois vértices opostos, mede $\alpha=\arccos \left(\frac{-1}{\sqrt{5}}\right) \approx 116,565^{\circ}$. Esses dois vértices são aqueles de configuração $(6,6,6)$ (encontro de três hexágonos). Os outros quatro ângulos, correspondentes aos vértices de configuração $(5,6,6)$, medem $\beta \approx 121,717^{\circ}$. Para facilitar o desenho e o recorte dessas faces hexagonais calculamos sua altura: $H=\ell\left(2+\frac{2}{\sqrt{5}}\right)^{1 / 2} \approx 1,701 \ell$, sendo $\ell$ o lado do hexágono e H a distância entre os dois lados opostos contíguos a ângulos de medida $\beta$. Uma face hexagonal do TRT está desenhada na Figura 23. As medidas dos ângulos foram obtidas de [26].

Para facilitar o recorte das faces pentagonais regulares de lado $\ell$, informamos que sua altura (distância de um vértice ao lado oposto) é $\mathrm{h}=\frac{1}{2}(\sqrt{5+2 \sqrt{5}}) \ell \approx 1,539 \ell$.

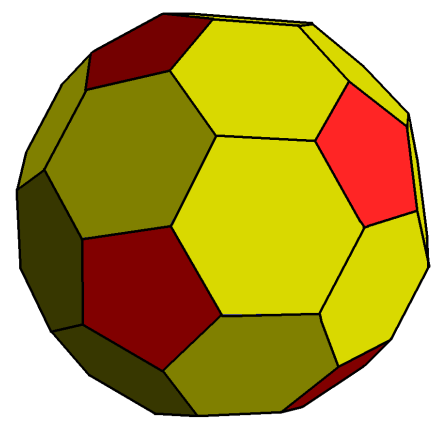

Figura 22: O poliedro de Goldberg triacontaedro rômbico truncado (TRT), um modelo da estrutura $\mathrm{C}_{80}$. Figura da Wikipedia [26].

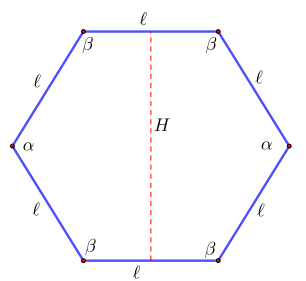

Figura 23: Face hexagonal do poliedro de Goldberg TRT. 
Figura 24. Modelo em papel-cartão do triacontaedro rômbico truncado, construído pelo autor. O molde das faces hexagonais, em cor laranja, é o da Figura 23, com $\ell=4 \mathrm{~cm}$. As faces pentagonais são regulares e estão em cor vermelha. O modelo está pousado em uma face pentagonal. Foto do autor.

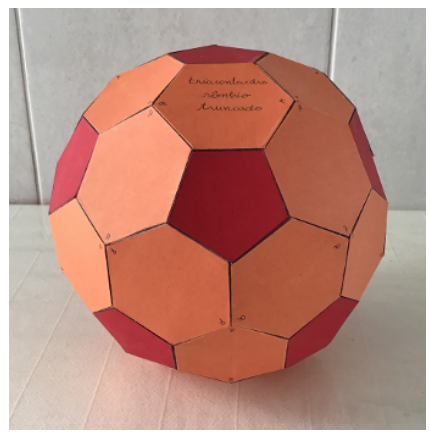

Notemos que no TRT, assim como no icosaedro truncado, cada face pentagonal está rodeada de um primeiro anel de cinco faces hexagonais, e de um segundo anel com 10 faces, sendo cinco pentagonais e cinco hexagonais. A diferença entre eles é que no TRT o segundo anel está rotacionado em relação ao mesmo anel do icosaedro truncado. Isso faz com que no TRT existam vértices rodeados por três faces hexagonais (portanto essas faces não podem ser regulares). Essa disposição no TRT implica que exista um terceiro anel com 10 faces hexagonais, o que não ocorre no outro poliedro. E essa é a diferença entre o icosaedro truncado e o TRT: o primeiro tem 32 faces, sendo 20 hexagonais, e o segundo, 42 faces, sendo 30 hexagonais.

\section{Algumas considerações sobre isômeros}

Vimos dois poliedros representantes da estrutura $\mathrm{C}_{60}$ : um é o icosaedro truncado, mostrado na Figura 3, e o outro é o fulereno-tubo, Figura 16. Vimos ainda dois representantes do $\mathrm{C}_{80}$ : o fulereno-tubo da Figura 18 e o poliedro de Goldberg da Figura 22. Duas representações da mesma estrutura se dizem isômeros.

Mais exatamente, chamamos de isômeros dois fulerenos com o mesmo número de faces hexagonais mas com as faces (pentágonos e hexágonos) dispostas em arranjos diferentes.

Dois isômeros, quando representam modelos de moléculas reais, formam compostos com propriedades químicas diferentes. Em particular, na estrutura $\mathrm{C}_{60}$, o icosaedro truncado é o único isômero que tem todos os pentágonos isolados entre si. Esse é um dos critérios relatados na literatura como importante para a estabilidade molecular (confira Corrêa e Reis [2], pág. 964).

A contagem dos isômeros exige técnicas especiais e é relativamente difícil, pois a quantidade de isômeros de $\mathrm{C}_{\mathrm{n}}$ aumenta exponencialmente em relação a $\mathrm{n}$. Vemos abaixo uma tabela, extraída de [5], mostrando, para alguns valores, a quantidade $\mathrm{I}$ de isômeros em relação a $\mathrm{C}_{\mathrm{n}}$ e a $\mathrm{F}_{6}$.

\begin{tabular}{|c|c|c|c|c|c|c|c|c|c|c|c|c|}
\hline $\mathrm{C}_{\mathrm{n}}$ & $\mathrm{C}_{20}$ & $\mathrm{C}_{24}$ & $\mathrm{C}_{26}$ & $\mathrm{C}_{28}$ & $\mathrm{C}_{30}$ & $\mathrm{C}_{32}$ & $\mathrm{C}_{34}$ & $\mathrm{C}_{36}$ & $\ldots$ & $\mathrm{C}_{60}$ & $\ldots$ & $\mathrm{C}_{170}$ \\
\hline $\mathrm{F}_{6}$ & 0 & 2 & 3 & 4 & 5 & 6 & 7 & 8 & $\ldots$ & 20 & $\ldots$ & 75 \\
\hline $\mathrm{I}$ & 1 & 1 & 1 & 2 & 3 & 6 & 6 & 15 & $\ldots$ & 1812 & $\ldots$ & 46.088 .157 \\
\hline
\end{tabular}

Vejamos como é o segundo isômero do $\mathrm{C}_{28}$ (confira no artigo de Fowler e Austin [4], pág. 265).

Uma forma de diferenciar os dois isômeros da estrutura $\mathrm{C}_{28}$ consiste em examinar a configuração de seus vértices. Um vértice diz-se ter a configuração $(\mathrm{m}, \mathrm{n}, \mathrm{p})$ quando estiver rodeado por um polígono com m lados, outro com n lados, e o terceiro com p lados. 
Quanto aos dois isômeros do $\mathrm{C}_{28}$, temos:

Isômero 1 do $\mathbf{C}_{28}$ : desenho na Figura 14 e foto na Figura 15. Ele tem 24 vértices com a configuração $(6,5,5)$ e 4 vértices com a configuração $(5,5,5)$.

Isômero $\mathbf{2}$ do $\mathbf{C}_{28}$ : desenho na Figura 25, planificação na Figura 26 e foto na Figura 27. Ele tem 16 vértices com a configuração $(6,5,5), 8$ vértices com a configuração $(5,5,5)$ e 4 vértices com a configuração $(6,6,5)$.

Entretanto, dois isômeros da mesma estrutura $\mathrm{C}_{\mathrm{n}}$ nem sempre têm configurações diferentes.

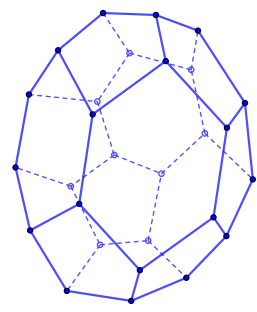

Figura 25: Representação do segundo isômero do $\mathrm{C}_{28}$.

Figura 27. Modelo do segundo isômero do $\mathrm{C}_{28}$, construído pelo autor em papel-cartão. Um desenho e uma planificação do mesmo poliedro estão nas figuras 25 e 26, respectivamente. As faces foram recortadas como polígonos regulares, o que causa uma pequena distorção. As faces hexagonais estão em cor vermelha, e as faces pentagonais em cor laranja. O modelo está pousado em uma face pentagonal.

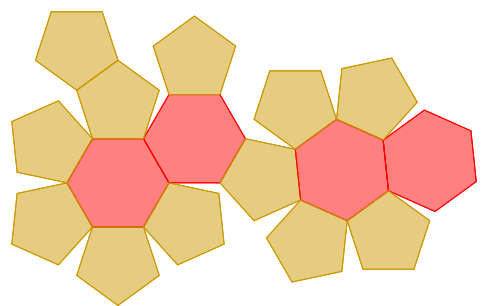

Figura 26: Planificação (indicativa) do segundo isômero do $\mathrm{C}_{28}$.

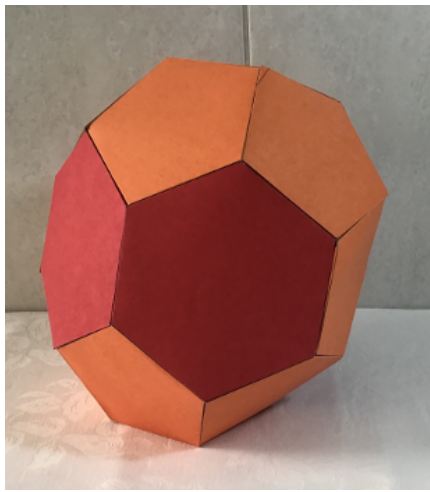

\section{Notas técnicas finais}

Reservamos, para o final deste texto, a exposição de algumas considerações técnicas, destinadas mais ao professor.

Nota 1. Comentários sobre a demonstração do Teorema 2 podem ser lidos no livro de Grünbaum [7], a partir da pág. 271. Entretanto, usando algumas técnicas, não é difícil convencermo-nos da validade desse Teorema. Consideremos as figuras 28-32, as quais adaptamos de Grünbaum [7], pág. 280, exercício 1.

$\mathrm{Na}$ Figura 28 vemos uma representação, na forma de um grafo, da "metade" superior do $\mathrm{C}_{24}$, conforme apresentado na Figura 8. Vemos um hexágono rodeado de seis pentágonos. Realizamos 
uma operação na parte central deste grafo, transformando o hexágono em dois pentágonos. Em compensação, dois dos antigos pentágonos transformam-se em hexágonos. Obtemos o grafo descrito na Figura 29, com seis pentágonos e dois hexágonos. Portanto, essa operação, que chamaremos de $\mathrm{T}_{1}$, conserva a quantidade de pentágonos e aumenta a de hexágonos em uma unidade.

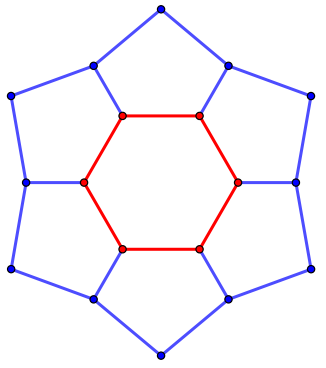

Figura 28: $\mathrm{F}_{5}=6, \mathrm{~F}_{6}=1$.

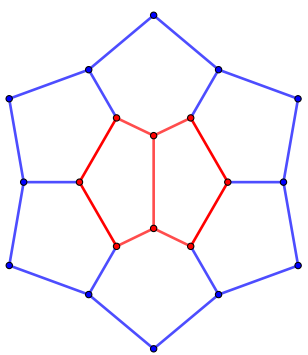

Figura 29: $\mathrm{F}_{5}=6, \mathrm{~F}_{6}=2$.

Se fizermos a operação $\mathrm{T}_{1}$ em uma metade do $\mathrm{C}_{24}$ obtemos o $\mathrm{C}_{26}$, e, se fizermos nas duas metades, obtemos o $\mathrm{C}_{28}$.

Consideremos agora a operação $\mathrm{T}_{2}$, ilustrada na Figura 30, que transforma o hexágono do grafo da Figura 28 em três pentágonos, e transforma três dos pentágonos externos em hexágonos. Portanto, a operação $\mathrm{T}_{2}$ conserva a quantidade de pentágonos e aumenta a de hexágonos em duas unidades.

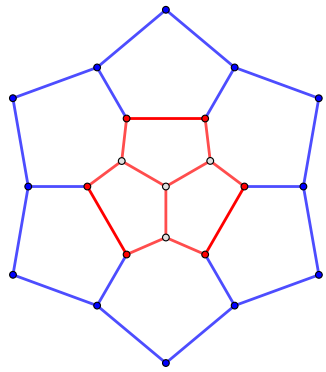

Figura 30: $\mathrm{F}_{5}=6, \mathrm{~F}_{6}=3$.

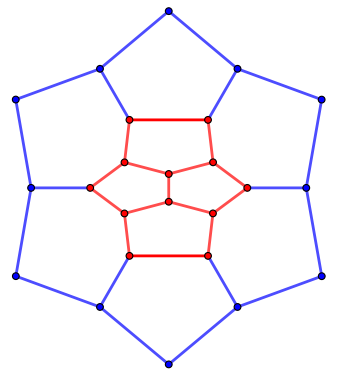

Figura 31: $\mathrm{F}_{5}=6, \mathrm{~F}_{6}=4$.

Se fizermos a operação $\mathrm{T}_{2}$ em uma metade do $\mathrm{C}_{24}$ obtemos o $\mathrm{C}_{28}$. Se fizermos a operação $\mathrm{T}_{1}$ em uma metade e a $\mathrm{T}_{2}$ na outra metade, obtemos o $\mathrm{C}_{30}$. Aplicando a operação $\mathrm{T}_{2}$ nas duas metades, obtemos o $\mathrm{C}_{32}$.

A operação $\mathrm{T}_{3}$ está descrita pela Figura 31. O hexágono do grafo da Figura 28 transforma-se em quatro pentágonos, e quatro dos pentágonos externos viram hexágonos. Assim, a operação $\mathrm{T}_{3}$ conserva a quantidade de pentágonos e aumenta a de hexágonos em três unidades. Se fizermos a operação $\mathrm{T}_{3}$ em uma metade do $\mathrm{C}_{24}$, obtemos o $\mathrm{C}_{30}$ (um isômero daquele obtido acima). No mesmo esquema, $\mathrm{T}_{1}$ com $\mathrm{T}_{3}$ gera $\mathrm{C}_{32}, \mathrm{~T}_{2}$ com $\mathrm{T}_{3}$ gera $\mathrm{C}_{34}$ e $\mathrm{T}_{3}$ com $\mathrm{T}_{3}$ gera $\mathrm{C}_{36}$. 
Figura 32. Descrição da operação $\mathrm{T}_{4}$. Ela consiste em rodear os quatro grafos anteriores com seis novos hexágonos, mantendo a forma da linha externa. Desse modo, reduzindo o desenho, podemos acoplá-lo com a segunda metade do $\mathrm{C}_{24}$ e também daqueles obtidos com a aplicação das operações anteriores. Na verdade, essa operação consiste em inserir uma cinta de seis hexágonos entre as duas partes de todos os fulerenos já construídos.

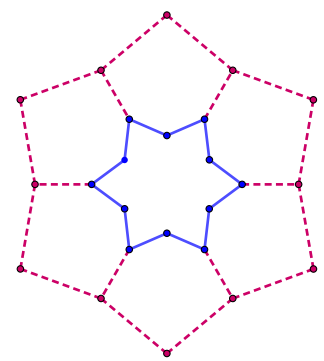

Aplicando a operação $\mathrm{T}_{4}$, descrita na Figura 32, aos fulerenos $\mathrm{C}_{24}$ a $\mathrm{C}_{36}$, obtemos, respectivamente, os fulerenos $\mathrm{C}_{36}$ a $\mathrm{C}_{48}$. Essa operação, por sua vez, pode ser repetida com esses últimos, de modo que obtemos todos os fulerenos $C_{n}$ para $n=20$ e $n \geq 24$ par. Assim, todos os valores inteiros $\mathrm{F}_{6} \geq 0$ são usados, exceto $\mathrm{F}_{6}=1$.

Observamos que, a partir da segunda cinta, temos fulerenos-tubo com diversas terminações nas capas de fechamento.

Por outro lado, para nos convencermos de que não existe fulereno com $\mathrm{F}_{6}=1$, podemos raciocinar a partir de tentativas de construir um modelo. Dispondo de 12 pentágonos e 1 hexágono recortados, começamos com este último. Ele tem que ser rodeado de 6 pentágonos. Como todo vértice é de tipo 3, esses pentágonos precisam estar conectados, cada um com seu vizinho, pois não é possível inserir outra face entre dois deles. Fica assim formada uma capa. Essa capa tem seis "entradas", que devem ser preenchidas com os seis pentágonos restantes, cada um dos quais precisa estar conectado com seu vizinho. Sobra uma abertura, e não temos mais faces para completá-la.

Nota 2. Comentamos, na Seção 3, que os fulerenos $\mathrm{C}_{24}, \mathrm{C}_{26}$ e $\mathrm{C}_{28}$ não podem ter todas as faces regulares. Em geral os únicos poliedros convexos com faces pentagonais ou hexagonais regulares são o dodecaedro regular e o icosaedro truncado semirregular. Podemos constatar isso observando que não são prismas e nem antiprismas, e apenas o dodecaedro regular e o icosaedro truncado semirregular fazem parte das famílias dos sólidos de Platão, de Arquimedes e de Johnson. Mais informações sobre essas famílias podem ser encontradas em [24].

Resta-nos explicar por que aparentemente dá certo usar faces regulares para construir modelos de alguns fulerenos, como o $\mathrm{C}_{24}$.

Primeiro observamos que se um poliedro é convexo e todos os seus vértices são do tipo três, então, os vértices formam triedros. Um triedro tem três ângulos planos, digamos $\alpha, \beta$ e $\gamma$, que são os ângulos do vértice correspondente das faces que a ele concorrem, e tem três diedros. A medida $[\alpha]$ do diedro oposto a $\alpha$ é dada pela fórmula

$$
\cos [\alpha]=\frac{\cos \alpha-\cos \beta \cos \gamma}{\operatorname{sen} \beta \operatorname{sen} \gamma}
$$

Com essa fórmula vemos, em particular, que todo triedro é rígido. Assim, se dois triedros tiverem ângulos planos de mesma medida, então os diedros correspondentes também têm a mesma medida.

Se um poliedro convexo tem faces exclusivamente pentagonais e hexagonais regulares, os ângulos das faces dos triedros (necessariamente todo vértice formará um triedro) medem $108^{\circ}$ ou $120^{\circ}$. $\mathrm{Na}$ tabela abaixo indicamos os triedros por $(5,5,5),(5,5,6)$ e $(5,6,6)$, sendo 5 correspondendo a uma 
face pentagonal e 6 , a uma hexagonal. No triedro $(5,5,6)$, o símbolo $[5,5]$ indica a medida do diedro oposto ao hexágono, e [5,6] a medida do diedro oposto ao pentágono, e assim por diante.

Usando a fórmula (8), podemos calcular as medidas de todos os diedros possíveis, como se vê na tabela abaixo.

\begin{tabular}{|c|c|c|c|}
\hline triedro & diedro 1 & diedro 2 & $\operatorname{diedro~} 3$ \\
\hline$(5,5,5)$ & {$[5,5] \approx 116,565051177^{\circ}$} & {$[5,5] \approx 116,565051177^{\circ}$} & {$[5,5] \approx 116,565051177^{\circ}$} \\
\hline$(5,5,6)$ & {$[5,5] \approx 131,174856819^{\circ}$} & {$[5,6] \approx 124,248093458^{\circ}$} & {$[5,6] \approx 124,248093458^{\circ}$} \\
\hline$(5,6,6)$ & {$[5,6] \approx 142,622631859^{\circ}$} & {$[5,6] \approx 142,622631859^{\circ}$} & {$[6,6] \approx 138,189685104^{\circ}$} \\
\hline
\end{tabular}

Vejamos agora por que o $\mathrm{C}_{24}$ não pode ter todas as faces regulares.

Figura 33. Escolhemos dois vértices contíguos $\mathrm{V}_{1}$ e $\mathrm{V}_{2}$ do poliedro $\mathrm{C}_{24}$, parte do desenho na Figura 8. $\mathrm{V}_{1}$ é um triedro do tipo $(5,5,6)$ e seu diedro comum com seu vizinho é $[5,5] \approx 131,174856819^{\circ}$. Por outro lado, $\mathrm{V}_{2}$ é um triedro do tipo $(5,5,5)$ e seu diedro comum com seu vizinho é $[5,5] \approx 116,565051177^{\circ}$. Consequentemente, se as faces forem regulares, os dois diedros não combinam.

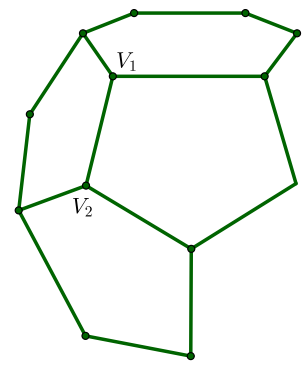

Vemos que situação semelhante ocorre com $\mathrm{C}_{26}, \mathrm{C}_{28}$, e outros.

Para completar, lembramos que se três hexágonos regulares (portanto são planos) encontram-se em um vértice (e se intersectam apenas em arestas), então eles formam uma figura plana. De fato, se formassem uma figura não plana, o referido vértice seria origem de um triedro. Nesse triedro a soma dos ângulos das faces seria $3 \times 120^{\circ}=360^{\circ}$. Mas a soma dos ângulos planos de um triedro deve ser $<360^{\circ}$. Para ver uma demonstração desse resultado consulte artigo de Paterlini [11].

Encerramos esta nota com a constatação de que a diferença das medidas dos diedros mencionados é de apenas 12,5\% em relação à medida menor, de forma que, se os modelos forem construídos com papel-cartão ou varetas, a distorção pode passar despercebida.

Nota 3. Uma breve explicação sobre o que são simetrias icosaédricas. Considere um icosaedro regular, e uma reta que passa por dois vértices opostos, como na Figura 34. As rotações em torno desse eixo com ângulos $72^{\circ}, 144^{\circ}, 216^{\circ}$ e $288^{\circ}$ deixam o icosaedro invariante, assim como a rotação de ângulo $0^{\circ}$, que é a identidade. Essas rotações são simetrias do icosaedro regular. Como o icosaedro tem 12 vértices que se organizam em seis pares de vértices opostos, temos seis eixos que dão lugar a $6 \times 4=24$ simetrias, e mais a identidade.

Consideremos agora as retas que passam pelo centro de duas faces opostas do icosaedro (um exemplo está desenhado na Figura 35). Como existem 20 faces que formam 10 pares de faces opostas, temos 10 eixos nessas condições. As rotações de $120^{\circ}$ e de $240^{\circ}$ que fixam esses eixos deixam o icosaedro invariante. Assim, temos mais $10 \times 2=20$ simetrias. 


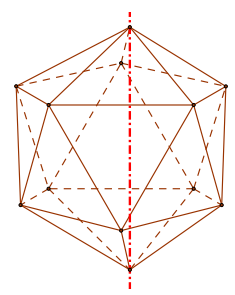

Figura 34: Eixo por dois vértices opostos.

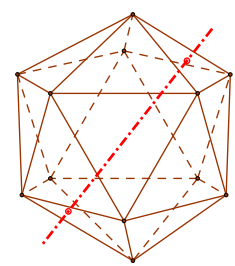

Figura 35: Eixo por duas faces opostas.

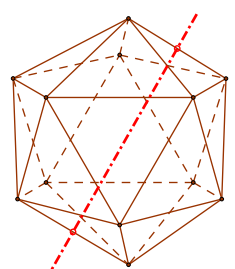

Figura 36: Eixo por duas arestas opostas.

Por fim, consideremos a mediatriz de duas arestas opostas (Figura 36). Uma rotação de $180^{\circ}$ que fixa esse eixo deixa o icosaedro invariante. Como são 30 arestas, existem 15 desses eixos, que determinam mais 15 simetrias.

Contando com a identidade temos $1+24+20+15=60$ simetrias. Essas simetrias formam uma estrutura matemática de grupo, nesse caso denominado grupo icosaédrico.

Dizemos que tem simetria icosaédrica qualquer poliedro com simetrias que formam um grupo com a mesma estrutura do grupo icosaédrico. Exemplos de poliedros com simetria icosaédrica, além do icosaedro regular, são o dodecaedro regular e o icosaedro truncado semirregular.

Nota 4. Uma dica, que usei em alguns casos, para a construção de modelos. Primeiro recortei 12 faces pentagonais regulares, e depois uma boa quantidade de faces hexagonais regulares. Usei papel-cartão de cores diferentes para os dois tipos de faces. As faces foram recortadas com abas de $1 \mathrm{~cm}$ de largura. Para juntar faces, usei grampos, como se vê na Figura 37. Os grampos são presos internamente até o ponto em que isso seja possível e externamente no final da montagem. Dessa forma os mesmos recortes de faces podem ser reaproveitados para vários modelos e para investigação de isômeros.

Figura 37. Um modelo com grampos expostos, construído em papel-cartão pelo autor. Foto do autor.

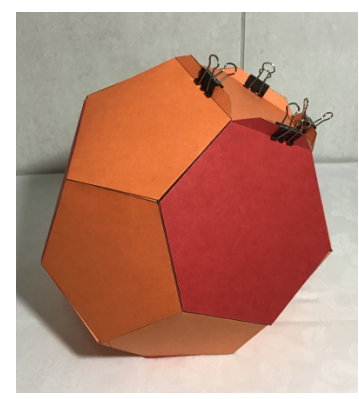

\section{Agradecimentos}

O autor agradece ao Prof. Romeu C. Rocha-Filho, do Departamento de Química da UFSCar, que leu o original e fez sugestões que levaram ao seu aprimoramento. 


\section{Referências}

[1] Buchstaber, V. M. e Erokhovets, N. Y. Fullerenes, Polytopes and Toric Topology. Versão publicada no Arxiv: https://arxiv.org/abs/1609.02949 Consultado em 23 de janeiro de 2020.

[2] Corrêa, T. H. B. e Reis, J. D. S. Estruturas de fulerenos: estabelecendo interfaces no ensino de Matemática e Química em nível superior. Quim. Nova, Vol. 40, No. 8, 963-969, 2017. http: //dx.doi.org/10.21577/0100-4042.20170053

[3] Cox, B. J. e Hill, J. M., Exact and approximate geometric parameters for carbon nanotubes incorporating curvature. Carbon 45 (2007) 1453-1462. doi:10.1016/j.carbon.2007.03.028

[4] Fowler, P. W. e Austin, S. J. Closed-Shell Carbon Frameworks: Leapfrog Fullerenes and Decorated Spheriphane Hydrocarbons. J. Chem. Inf. Comput. Sci. 1994, 34, 264-269.

[5] Goedgebeur, J. e McKay, B. D. Recursive generation of IPR fullerenes. J Math Chem v. 53, pp. 1702-1724, 2015.

[6] Goldberg, M. (1937). A class of multi-symmetric polyhedra. Tohoku Math J v. 43, pp.104, 1937.

[7] Grünbaum, B. Convex Polytopes. Segunda edição. N. York: Springer, 2003.

[8] Hart, G. Goldberg Polyhedra. In Senechal, M. (ed.), Shaping Space: Exploring Polyhedra in Nature, Art, and the Geometrical Imagination. New York: Springer, 2013.

[9] Melker, A. I. e Krupina, M. A. Geometric modeling of midi-fullerenes growth from $\mathrm{C}_{24}$ to $\mathrm{C}_{48}$. St. Petersburg Polytechnical University Journal: Physics and Mathematics v. 2, pp.201-208, 2016. Obtido em https://www.sciencedirect.com/science/article/pii/S2405722316301074

[10] Parasuk, V. e Amlöf J. $C_{20}$ : the smallest fullerene? Chemical Physics Letters, v. 184, number 1, 2, 3, pp.187-190, 1991.

[11] Paterlini, R. R. Sobre a soma dos ângulos planos de ângulos poliédricos convexos. PMO v.8, n.2, 2020, 156-169. ISSN: 2319-023X https://doi.org/10.21711/2319023x2020/pmo812

[12] Paterlini, R. R. Geometria Elementar, gênese e desenvolvimento. Disponível em https://www. dm.ufscar.br/ ptlini/geometria_Paterlini_27_01_2020.pdf Acesso em: 18 de maio de 2020.

[13] Prinzbach, H. et alii Gas-phase production and photoelectron spectroscopy of the smallest fullerene, $\mathrm{C}_{20}$. Nature, Vol 407, 7 September 2000, pp.60-63. DOI: 10.1038/35024037

[14] Rocha-Filho, R. C. Os fulerenos e sua espantosa geometria molecular. Química Nova na Escola, n. 4, 1996. Disponível em: http://qnesc.sbq.org.br/online/qnesc04/atual.pdf

[15] Schein S. e Gayed, J. M. Fourth class of convex equilateral polyhedron with polyhedral symmetry related to fullerenes and viruses. Proc. Natl. Acad. Sci. USA 2014, v. 111, n. 8, pp.2920-2925.

[16] Schwerdtfeger, P., Wirz, L. N. e Avery, J. The topology of fullerenes. WIREs Comput Mol Sci 2015, 5:96-145. doi: 10.1002/wcms.1207

[17] Talanov, V. M. et alii Symmetrical Features of the Structure of $\mathrm{C}_{24}$ and $\mathrm{C}_{48}$ Fullerenes. Glass Physics and Chemistry, v. 36, No. 3, pp.358-368, 2010.

[18] Wikipedia. Dodecahedrane. Disponível em https://en.wikipedia.org/wiki/Dodecahedrane Consultado em 18 de janeiro de 2020.

[19] Wikipedia. Truncated icosahedron. Disponível em https://en.wikipedia.org/wiki/Truncated icosahedron Endereço da Figura 3: https://commons.wikimedia.org/wiki/File:Polyhedron truncated_20_max.png Autor: Watchduck. GNU Free Documentation License. Consultado em 20 de janeiro de 2020 . 
[20] Wikipedia. Buckminsterfullerene. Disponível em https://en.wikipedia.org/wiki/ Buckminsterfullerene Endereço da Figura 4: https://commons.wikimedia.org/wiki/File: Buckminsterfullerene.svg Autor: Leyo. Domínio público. Consultado em 20 de janeiro de 2020.

[21] Wikipedia. Buckminster Fuller. Disponível em https://en.wikipedia.org/wiki/Buckminster Fuller Consultado em 23 de janeiro de 2020.

[22] Wikipedia. Pentagonal icositetrahedron. Disponível em https://en.wikipedia.org/wiki/ Pentagonal_icositetrahedron Consultado em 24 de janeiro de 2020.

[23] Wikipedia. Dodecahedron. Disponível em https://en.wikipedia.org/wiki/Dodecahedron Consultado em 24 de janeiro de 2020.

[24] Wikipedia. Johnson solid. Disponível em https://en.wikipedia.org/wiki/Johnson_solid Consultado em 9 de fevereiro de 2020.

[25] Wikipedia. Goldberg polyhedron. Disponível em https://en.wikipedia.org/wiki/Goldberg polyhedron A Figura 21 foi adaptada de https://commons.wikimedia.org/wiki/File:Goldberg 10_0_equilateral-spherical.png Autor: Tomruen. Licença Creative Commons Attribution-Share Alike 4.0 International. Consultado em 9 de fevereiro de 2020.

[26] Wikipedia. Chamfered dodecahedron. Disponível em https://en.wikipedia.org/wiki/ Chamfered_dodecahedron A Figura 22 foi obtida de https://commons.wikimedia.org/wiki/File: Truncated_rhombic_triacontahedron.png Autor: Tomruen, domínio público. Consultado em 4 de abril de 2020 .

[27] Wikipedia. Fullerene. Disponível em https://en.wikipedia.org/wiki/Fullerene Endereço da Figura 1: https://commons.wikimedia.org/wiki/File:C20_Fullerene.png Autor: Perditax. Data: 11 de junho de 2011. Domínio público. Consultado em 19 de agosto de 2020.

Roberto Ribeiro Paterlini Universidade Federal de São Carlos $<$ paterlini@ufscar.br>

Recebido: 29/05/2020 Publicado: 24/09/2020 